Une source de lumière

de dimension atomique

est réalisée à l'aide d'un

microscope à effet tunnel (STM).

Cette source de photons est

localisée à la jonction entre

une pointe, que l'on peut

déplacer avec une précision

atomique, et un échantillon

métallique. Elle se distingue

par une excitation de nature

électronique (le courant tunnel),

et fait également intervenir des

plasmons de surface présents

dans la jonction tunnel.

La jonction tunnel est ainsi

une « source électrique » de

plasmons de surface.

Cependant, on est encore

loin de comprendre le

fonctionnement de cette

source optique et plasmonique

et d'en avoir exploité toutes

les possibilités.

\title{
Émission de lumière sous la pointe d'un microscope à effet tunnel
}

Guillaume Schull(1) (guillaume.schull@ipcms.unistra.fr), Élizabeth Boer-Duchemin $^{(2)}$,

Geneviève Comtet ${ }^{(2)}$ et Gérald Dujardin ${ }^{(2)}$

(1) Institut de Physique et Chimie des Matériaux de Strasbourg, UMR 7504 (CNRS - Université de Strasbourg),

67034 Strasbourg

(2) Institut des Sciences Moléculaires d’Orsay (ISM0), CNRS Université Paris-Sud, 91405 Orsay

En 1976, J. Lambe et S.L. McCarthy rapportent une découverte étonnante. Appliquant une différence de potentiel aux bornes d'une jonction tunnel macroscopique (deux électrodes métalliques séparées par une fine couche d'oxyde), ils détectent une émission de lumière. Ils remarquent que la couleur de cette émission évolue avec la tension appliquée V. Les chercheurs notent deux aspects essentiels : (i) l'énergie des photons émis ne dépasse pas celle des électrons de la jonction tunnel, de l'ordre de l'électron-volt ; (ii) le spectre d'émission dépend de la nature des électrodes, respectivement de l'aluminium et de l'or ou de l'argent. Les auteurs suggèrent que les photons sont créés par des électrons qui perdent de l'énergie au passage tunnel à travers la jonction, et excitent des oscillations collectives du gaz d'électrons des deux électrodes métalliques (plasmons de surface). Que se passe-t-il si l'on essaie de reproduire cet effet alors que la dimension de l'interface métal-isolant-métal est réduite à l'échelle nanométrique ? C'est chose faite en 1988, quand les chercheurs d'IBM positionnent la pointe d'un microscope à effet tunnel (STM) sur une surface d'argent et détectent l'émission lumineuse issue de la jonction "pointe métallique - vide - surface d'argent ».

Dans cet article, nous rapportons des expériences récentes qui illustrent la subtilité des phénomènes liés à l'émission de lumière dans la jonction tunnel du STM. Ces expériences illustrent tout d'abord la résolution spatiale ultime atteinte par cette source optique excitée électriquement.
En effet, des variations du spectre de l'émission lumineuse sont observées pour des modifications à l'échelle atomique de la position de la pointe STM par rapport à la surface de l'échantillon. D'autre part, ce sont des excitations collectives du métal, les plasmons de surface (voir encadré, p. 7), qui conduisent à l'observation de photons en champ lointain : la nature et le mode de rayonnement de ces plasmons est une question clé. Nos expériences montrent que certains modes de plasmons se propagent très loin (plusieurs dizaines de microns) sur la surface, alors que d'autres restent localisés sous la pointe STM. La forme et le matériau de la pointe permettent de contrôler le type de plasmons effectivement activés. Enfin, une seconde question cruciale est de comprendre comment les électrons du courant tunnel issus de la pointe du STM peuvent céder une partie de leur énergie et exciter les modes plasmons de la jonction tunnel. Une expérience récente suggère que ce sont les fluctuations temporelles du courant au passage de la jonction tunnel qui permettent l'excitation des plasmons et l'émission de photons.

\section{Une nanosource de photons...}

Le microscope à effet tunnel (STM) est un instrument emblématique de l'ère "nano ": il permet de réaliser des cartographies de surfaces conductrices avec une résolution atomique. Pour cela, une pointe métallique ultrafine est approchée à environ un nanomètre $(\mathrm{nm})$ de la surface. 


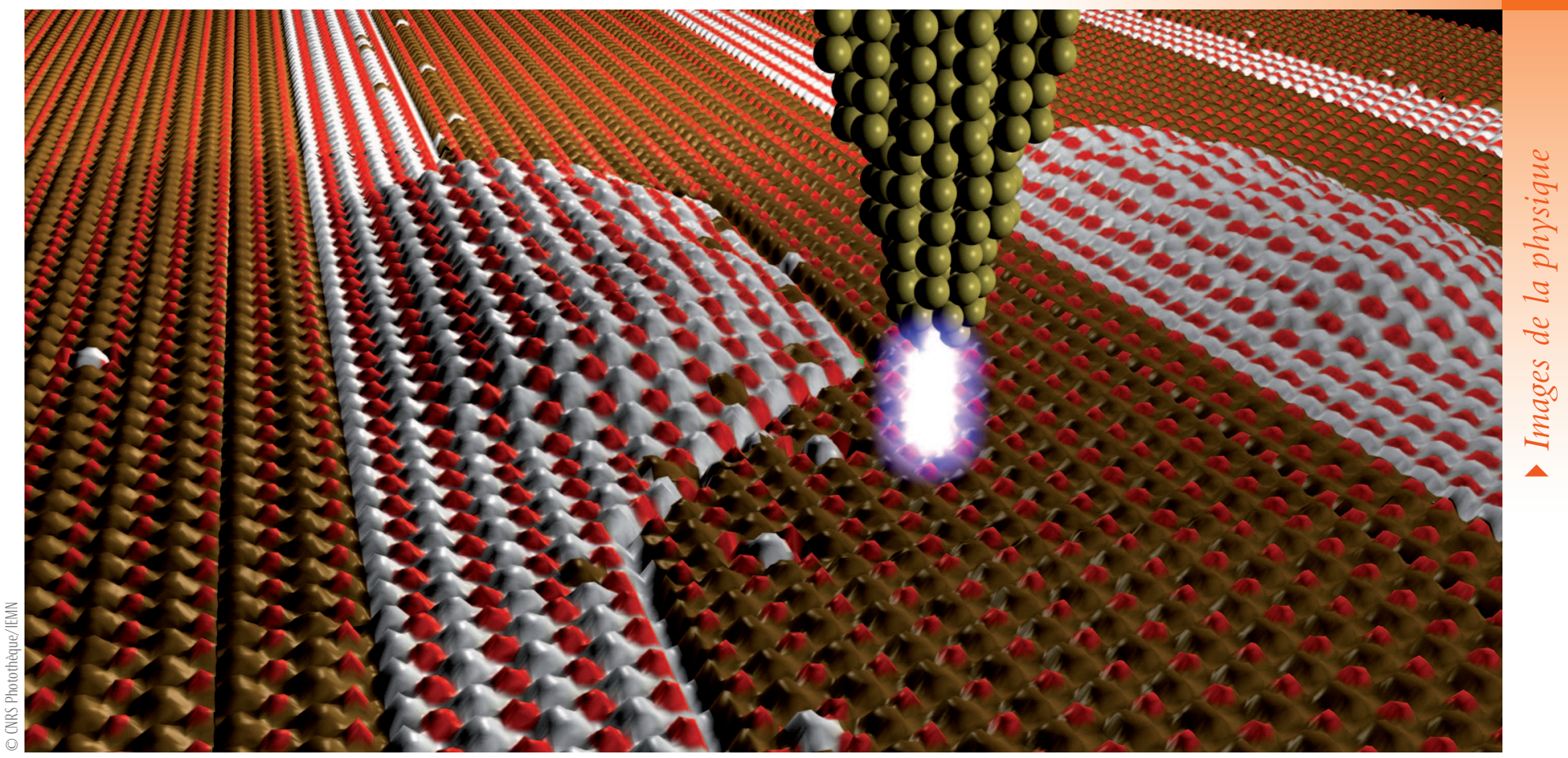

Image de synthèse d'une hétérostructure de semi-conducteur, accessible à la pointe d'un microscope à effet tunnel, suite au clivage de l'échantillon.

Lorsqu'une différence de potentiel ( 0 à $4 \mathrm{~V})$ est appliquée entre les deux électrodes, des électrons traversent la jonction pointesurface par effet tunnel. Un courant tunnel s'établit, dont l'intensité décroit exponentiellement avec la distance pointe-surface. En mesurant les variations de courant lors du balayage de la surface par la pointe, une cartographie de résolution atomique est obtenue. Le contrôle des vibrations et la précision des positionneurs piézoélectriques ont permis le développement de cet instrument extraordinaire, qui valut le prix Nobel à ses inventeurs, Binnig et Rohrer, en 1986. Comme dans les premières expériences, une émission de lumière peut être observée lors du passage des électrons tunnels entre pointe et échantillon métallique. L'intensité lumineuse est généralement faible, de l'ordre de $10^{-3}$ photons/électron pour des conditions " usuelles " de fonctionnement du STM $(I=1 \mathrm{nA}, V=2,5 \mathrm{~V})$. Nous sommes ici dans un régime de "comptage de photons ", et cette mesure nécessite la mise au point de dispositifs expérimentaux permettant de collecter la plus grande quantité de lumière émise.

Deux types de dispositifs sont utilisés. Dans le premier (fig. 1a), la jonction est positionnée au point focal d'une lentille collectrice située dans le même demiespace que la pointe. Le faisceau collecté est refocalisé (à l'extérieur d'un bâti à ultravide, par exemple) et mesuré à l'aide d'un détecteur de photons (compteur de photons, caméra CCD...). L'intensité, le spectre, la polarisation ou la statistique
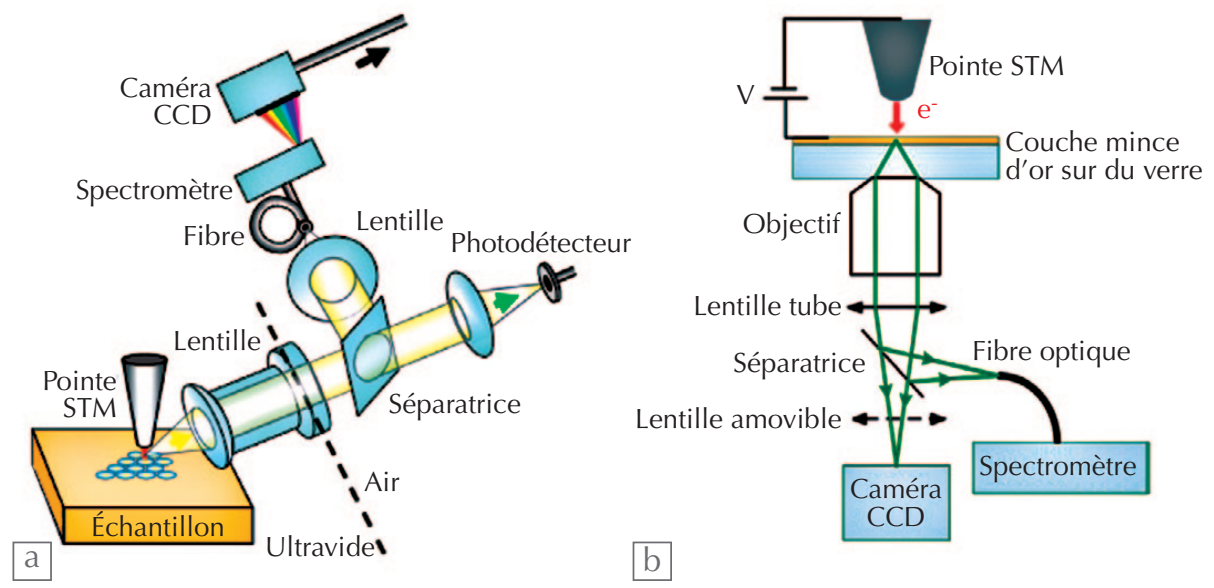

1. Détection de photons émis à une jonction STM :

(a) du côté de la pointe;

(b) par transmission à travers l'échantillon constitué d’un film mince d'or déposé sur une lamelle de verre.

d'émission des photons sont autant de paramètres qui peuvent être sondés. Ces paramètres vont évidemment varier en fonction du courant tunnel, de la tension appliquée, de la position de la pointe ou encore de la nature de l'objet sondé. On entrevoit ici toute la richesse d'un dispositif permettant de combiner les performances en termes de résolution spatiale du STM à des expériences d'optique. Mais seule l'émission de photons provenant du rayonnement de plasmons localisés dans la jonction tunnel peut être détectée ici. Les plasmons de surface propagatifs ne peuvent pas être détectés dans cette configuration, car leur courbe de dispersion ne croise pas celle des photons dans l'air (voir encadré, p. 7).
Un deuxième dispositif (fig. 1b) est donc nécessaire pour détecter simultanément les émissions de photons provenant de plasmons de surface localisés sous la pointe du STM et de plasmons propagatifs. Dans ce cas, le STM est monté au-dessus d'un microscope optique inversé. Cela permet la détection de lumière à travers un échantillon de faible épaisseur, conducteur et transparent, par exemple un film d'or déposé sur une lamelle de verre. Ici, on peut détecter, à l'aide d'une caméra CCD, l'image du plan objet (distribution spatiale) et l'image du plan de Fourier (distribution angulaire) de l'émission de photons. Avec ce dispositif, le STM peut être utilisé à l'air ou en atmosphère contrôlée (par exemple de l'azote), ainsi que dans un liquide. 


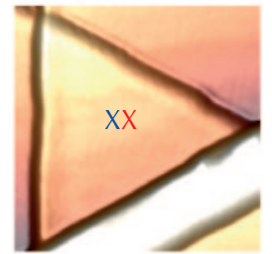

a b

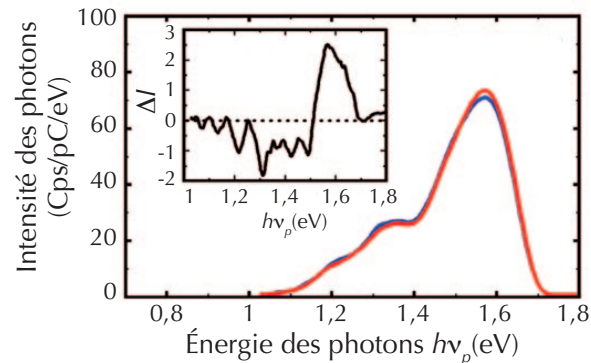

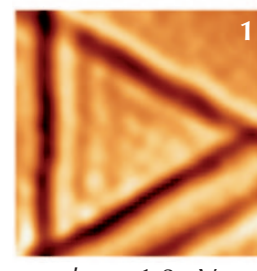

$h v_{p} \sim 1,3 \mathrm{eV}$

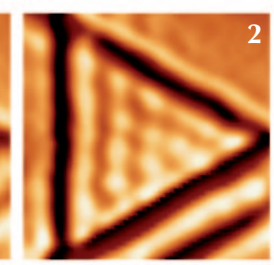

$h v_{p} \sim 1,5 \mathrm{eV}$

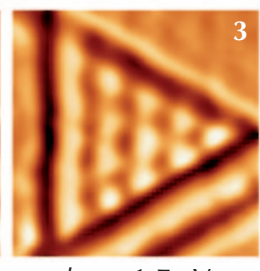

$h v_{p} \sim 1,7 \mathrm{eV}$
$v_{p}(\mathrm{eV})$

d

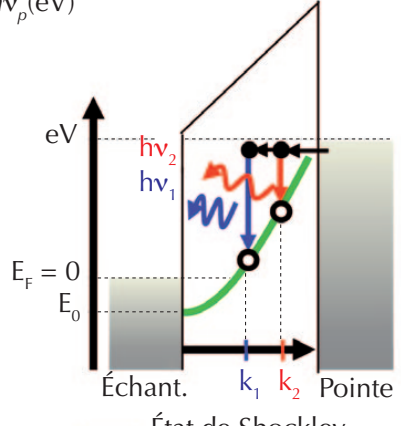

État de Shockley
2. Émission de lumière à la jonction entre une pointe STM et une nanostructure d'or.

(a) Image STM (11 x $\left.11 \mathrm{~nm}^{2}\right)$ d'un ilot triangulaire d'or sur une surface d'Au(111).

(b) Spectres optiques (en rouge et bleu) obtenus pour deux positions différentes de la pointe (croix rouge et bleue en (a)) face à cet ilot $\left(V_{s}=1,73 \mathrm{~V}, \mathrm{I}_{\mathrm{t}}=300 \mathrm{nA}\right)$. L'insert en (b) montre la différence entre ces deux spectres. (c) Cartes de photons obtenues en intégrant, pour chaque position de la pointe, les photons émis entre

(1) 1,25 eV $<\mathrm{h} v_{\mathrm{p}}<1,35 \mathrm{eV}$; (2) 1,45 eV $<\mathrm{h} v_{\mathrm{p}}<1,5 \mathrm{eV}$; et (3) 1,65 eV $<\mathrm{h} v_{\mathrm{p}}<1,7 \mathrm{eV}$.

(d) Modèle du mécanisme d'émission (voir texte).

\section{\〉}

\section{Le mécanisme de l'émission lumineuse}

Le processus d'émission de photons à la pointe d'un STM est complexe et certains de ses aspects sont encore mal compris. Une approche très simplifiée consiste à représenter ce processus par une expression dérivée de la règle d'or de Fermi, qui décrit le taux d'émission d'un émetteur quantique dans un état excité couplé à un continuum d'états :

$\Gamma_{\mathrm{rad}}=\left(2 \pi / \hbar^{2}\right) \mu_{\mathrm{if}}^{2} \rho\left(\omega=\omega_{\mathrm{if}}\right) E_{Z}(M)^{2}(1)$, où $\Gamma_{\text {rad }}$ est le taux d'émission, $\mu_{\text {if }}$ l'élément de matrice de l'opérateur moment dipolaire électrique pour une transition entre un état initial $(i)$ de la pointe et un état final $(f)$ de la surface, $\rho\left(\omega=\omega_{\text {if }}\right)$ est la densité spectrale de modes électromagnétiques à l'énergie de la transition $\left(\omega=\omega_{\mathrm{if}}\right)$, et $E(M)$ est la composante spatiale du champ électrique $E$ au point $M$ du système.

On voit ainsi que le rendement d'émission à une énergie donnée dépend de deux termes : un terme d'excitation, qui apparaît sous la forme de l'opérateur moment dipolaire $\mu_{\text {if }}$ et qui rend compte de la structure électronique du système, et un terme d'amplification correspondant aux composantes spectrales $\rho\left(\omega=\omega_{\text {if }}\right)$ et spatiales $E(M)$ du champ électromagnétique local.

Nous rapportons ici des expériences récentes, mettant en évidence le rôle (i) de la densité d'états électroniques de la surface, et (ii) des modes électromagnétiques de la jonction tunnel tels qu'ils apparaissent dans la règle d'or de Fermi. Nous discutons également d'autres expériences qui suggèrent que les fluctuations du courant tunnel, non prises en compte dans la règle d'or de Fermi, jouent un rôle essentiel dans l'émission de photons.

\section{Le rôle des états électroniques...}

Il s'agit ici d'une expérience qui met en évidence le rôle de l'état électronique local de la surface d'or sous la pointe STM dans le processus d'émission de lumière. Les spectres optiques reportés en bleu et rouge en figure $2 b$, ont été enregistrés pour deux positions différentes d'une pointe STM placée en regard d'une nanostructure triangulaire d'or apparaissant à la surface d'un échantillon de $\mathrm{Au}(111)$ (image STM, fig. 2a). Bien que les conditions expérimentales soient identiques, on note une légère variation des spectres en fonction de la position de la pointe (illustrée en insert de la figure 2b). Afin de mieux comprendre l'origine de ces différences, nous avons enregistré des spectres simultanément à l'acquisition de cartes topographiques. Ainsi, pour chaque pixel de l'image STM en (a), un spectre optique est enregistré, et il est alors possible de reconstruire de véritables "cartes de photons " $(64 \times 64$ pixels $)$ pour différentes longueur d'ondes des photons émis (fig. 2c). Ces cartes ont, dès lors, la même résolution spatiale que les images STM ! Elles montrent très nettement la présence d'ondes stationnaires au sein de la nanostructure, dont la périodicité varie avec l'énergie des photons (variation du nombre de nœuds d'une carte à l'autre). Ces ondes stationnaires sont caractéristiques du confinement latéral d'électrons localisés à la surface de la nanostructure. Comment expliquer que cet état de surface purement électronique puisse influencer le signal optique ?

Une étude détaillée permet de mettre en évidence les mécanismes régulant ici l'émission de lumière. Schématisé en figure $2 \mathrm{~d}$, le processus d'émission de photons est directement lié à des électrons injectés depuis le niveau de Fermi de la pointe, qui perdent une partie de leur énergie lors de leur passage vers la surface (flèches bleue et rouge). Dans le cas présent, ces électrons " inélastiques » terminent leurs parcours dans les états électroniques de surface de la nanostructure triangulaire. Pour une surface atomiquement plate et en l'absence de défauts, la courbe de dispersion de ces états (dans le plan $\mathrm{k}_{\mathrm{x}}, \mathrm{k}_{\mathrm{y}}$ ) est une parabole que nous avons schématisée en vert au centre de la jonction en figure $2 \mathrm{~d}$. On voit sur ce schéma que l'énergie $h v_{\mathrm{p}}$ des photons dépend directement du nombre d'onde $k$ de l'état d'arrivée, qui caractérise la périodicité des ondes électroniques observées dans les cartes de photons en figure $2 c$.

Cette expérience met en évidence différents aspects des processus d'émission de photons. Elle démontre notamment que les photons sont émis par des électrons tunnel « inélastiques » traversant la jonction. Conséquemment, la luminescence est fortement influencée par les états électroniques des objets localisés à la surface de l'échantillon. Cet effet est également à l'origine de la résolution atomique qui peut être observée au sein des cartes de photons. 


\section{... et celui des modes plasmoniques}

Compte tenu des dimensions nanométriques du bout de la pointe STM et de la jonction tunnel pointe-surface (bien plus petites que la longueur d'onde des photons, $\lambda \sim 700 \mathrm{~nm}$ ), les modes électromagnétiques les plus susceptibles d'être excités par un courant tunnel dans le modèle de la règle d'or de Fermi sont des modes plasmons (voir encadré). Il existe tout d'abord des plasmons localisés correspondant à des oscillations de la densité électronique dans la pointe et le film d'or, couplées à un champ électromagnétique ; d'autre part, des plasmons propagatifs, de vecteur d'onde $k_{S P P}$ parallèle à la surface du film d'or et de symétrie radiale, peuvent également être excités par le courant tunnel.

La question posée est donc de savoir quels sont les modes, plasmons localisés ou plasmons propagatifs, qui sont préférentiellement excités par le courant tunnel. Une expérience récente, utilisant le dispositif expérimental de la figure $1 \mathrm{~b}$, a permis de visualiser en deux dimensions (2D) le plan objet de l'émission de lumière créée par la pointe STM sur un film d'or de faible épaisseur $(50 \mathrm{~nm})$ déposé sur une lamelle de verre (fig. 3a). On y voit, d'une part, une émission très localisée sous la pointe, dont l'extension latérale, de l'ordre de $2 \mu \mathrm{m}$, est déterminée par la limite de diffraction du microscope optique et, d'autre part, une émission de lumière qui s'étend beaucoup plus loin du centre de l'image où se trouve la pointe STM (fig. 3b). Ces deux composantes de l'émission de lumière proviennent respectivement de plasmons localisés et de plasmons propagatifs. La longueur de propagation $L$ de ces derniers est estimée à environ 20 micromètres (fig. 3c).

Il est également observé que l'intensité relative de chaque mode de plasmon dépend de la géométrie et du matériau de la pointe STM. Avec une pointe fine en tungstène, on produit principalement des plasmons propagatifs, alors qu'avec une pointe en argent (qui possède une résonance plasmonique dans le visible) l'intensité des plasmons localisés est particulièrement élevée. On peut choisir ainsi de favoriser un type ou l'autre de mode plasmonique suivant que l'on veut augmenter localement le champ électromagnétique (plasmons localisés) ou bien faire du transfert d'information (plasmons propagatifs). On notera qu'avec le STM on produit des plasmons avec un spectre large en longueur d'onde
Un plasmon polariton de surface ("surface plasmon polariton" ou SPP) est une oscillation collective dans la direction de propagation $x$, couplée à un champ électromagnétique, des électrons à l'interface entre un métal et un diélectrique (fig. E1a). Un SPP propagatif se propage le long de cette interface et est évanescent dans la direction perpendiculaire à sa direction de propagation (fig. E1b), ce qui souligne le fait qu'il s'agit bien d'un mode localisé à la surface. La relation de dispersion (l'énergie ou la fréquence en fonction du vecteur $d^{\prime}$ onde $k_{S P P}$, où $k_{S P P}=2 \pi / \lambda$ et $\lambda$ est la longueur d'onde) pour un SPP à l'interface air-métal est représentée en figure $\mathrm{E} 2 \mathrm{a}$, ainsi que la relation de dispersion pour un photon dans l'espace libre et dans un matériau d'indice de réfraction $n>1$ (par ex. du verre). Le fait que les courbes de dispersion du SPP et du photon dans I'air ne se croisent pas (fig. E2a) implique que les SPPs sont non radiatifs et ne peuvent pas se coupler dans l'air avec des photons qui pourraient ensuite être détectés. Par contre, la courbe de dispersion d'un photon dans du verre croise bien la courbe de dispersion d'un SPP à l'interface airmétal (fig. E2a). Ainsi, une façon de détecter les plasmons propagatifs est de déposer une couche mince $(\leq 70 \mathrm{~nm})$ de métal sur du verre, d'exciter les SPP à l'interface air-métal, puis de collecter les photons émis à travers l'échantillon (dans le verre) (fig. E2b).

En revanche, les plasmons de surface localisés ou "localized surface plasmons", des oscillations collectives des électrons dans un espace confiné, comme par exemple une particule métallique de 2 à $20 \mathrm{~nm}$ de diamètre (fig. E3), se couplent facilement aux photons dans tout milieu diélectrique (air, verre...). Les plasmons localisés excités par une pointe STM sur une couche mince métallique sur du verre peuvent donc être détectés soit au-dessus (fig. 1a), soit au-dessous (fig. 1b) de l'échantillon, mais les plasmons de surface propagatifs ne peuvent être détectés qu'au-dessous de l'échantillon dans un matériau de plus fort indice de réfraction.

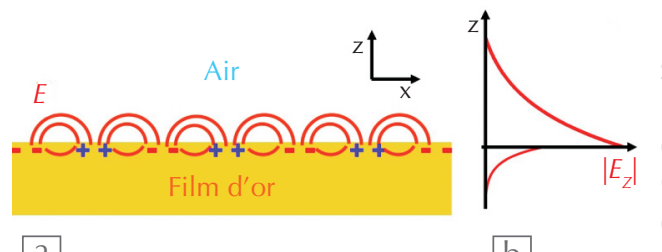

E1. (a) Schéma d'un plasmon polariton de surface (SPP) à l'interface entre un film d'or et l'air. Les arcs de cercles représentent les lignes de champ électrique.

(b) La composante $\mathrm{E}_{z}$ (perpendiculaire à l'interface) du champ électromagnétique lié au SPP est évanescente dans le film d'or et dans l'air.

a
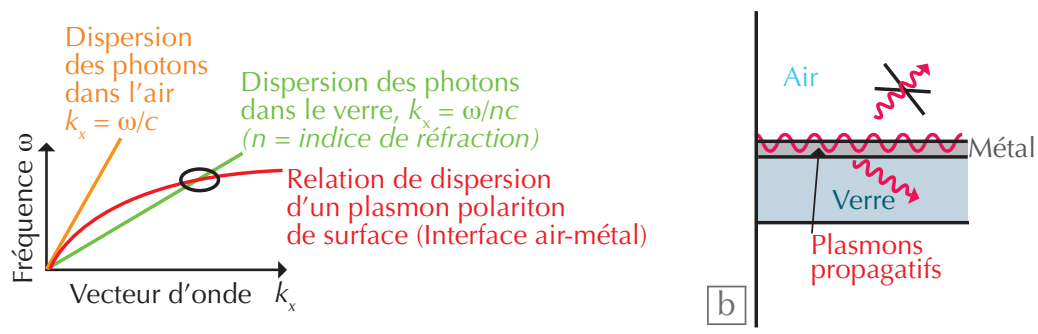

E2. (a) Relations de dispersion des photons dans l'air (en orange), des photons dans le verre (en vert) et des plasmons polaritons (propagatifs) de surface à l'interface air-métal (en rouge),

(b) Schéma montrant que l'égalité de la composante $k_{x}$ du vecteur d'onde du photon et du plasmon polariton de surface n'est possible que du côté du verre et pas du côté de l'air.

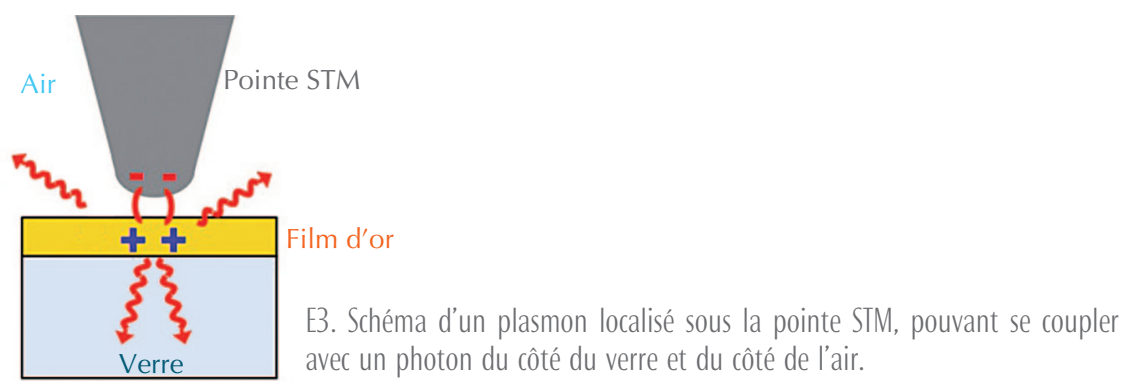




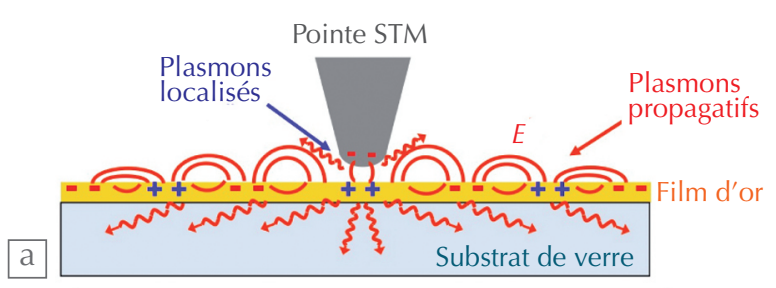

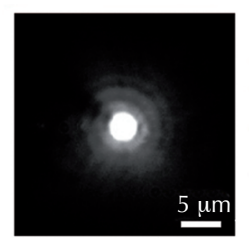

b

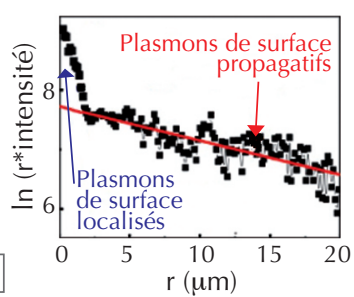

C

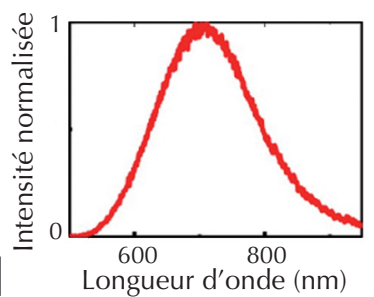

3. Émission de lumière et plasmons de surface.

(a) Schéma d'excitation des modes plasmons de surface localisés et propagatifs sous la pointe STM. Les arcs de cercles représentent les lignes de champ électrique des plasmons à l'interface, qui s'étendent à la fois du côté de l'air et du côté du film d'or (en jaune). Les flèches ondulées représentent les émissions de photons provenant des plasmons localisés et des plasmons propagatifs.

(b) Image du plan objet de l'émission de lumière sous la pointe STM, intégrée pendant 60 secondes.

(c) Coupe radiale de l'image (b), montrant l'émission des plasmons de surface localisés, et celle des plasmons de surface propagatifs qui s'étend jusqu'à plus de $20 \mu \mathrm{m}$ du centre de l'image où se trouve la pointe STM. On représente ici le logarithme du produit de l'intensité lumineuse par la distance (r) au centre.

(d) Spectre en longueur d'onde de lémission de lumière sous la pointe STM, observée à travers un film mince d'or (50 nm).
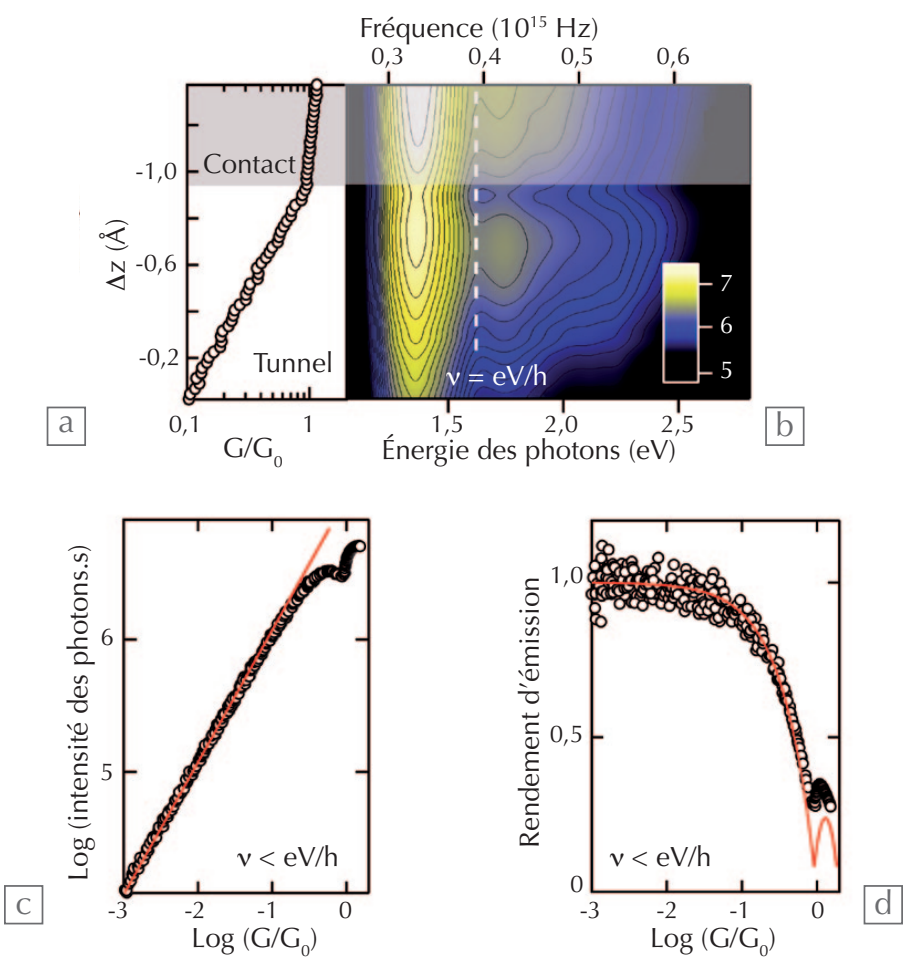

4. Variation de l'émission de lumière en fonction de la distance entre la pointe STM et la surface de l'échantillon.

(a) Conductance (dérivée du courant tunnel par rapport à la tension appliquée $V_{\text {ech) }}$ ) enregistrée lors de l'approche d'une pointe STM en argent d'un échantillon d'Ag(111), à partir des conditions tunnel $(\Delta z=0)$ jusqu'à un contact avec un atome (zone grisée) $\left(V_{\text {ech }}=1,6 \mathrm{~V}\right)$.

(b) Spectres de la lumière émise, enregistrés pour chaque incrément de l'approche en (a) (60 spectres). L'intensité des photons (coups/s/eV) est représentée en fausses couleurs sur une échelle logarithmique.

(c, d) Intensité des photons émis (c) et rendement d'émission (d) en fonction de la conductance de la jonction (cercles blancs) pour les photons ayant une énergie inférieure à celle des électrons de la jonction tunnel (1,6 eV). La ligne rouge correspond à une relation linéaire (c) ou au comportement du bruit de grenaille (d).

\section{\)}

(figs $3 \mathrm{~d}$ et $4 \mathrm{~b}$ ). Ceci peut être un avantage pour exciter simultanément plusieurs modes de plasmons ayant des longueurs d'ondes différentes.

\section{Fluctuations du courant tunnel}

Jusqu'ici nous nous sommes limités à des expériences d'émission de photons en régime tunnel, pour lesquelles une distance de $\sim 1 \mathrm{~nm}$ entre pointe et surface est considérée. Récemment, nous nous sommes intéressés à l'évolution des propriétés d'émission lors de la réduction de cette distance, puis de la fermeture de la jonction. Pour cela, nous avons approché une pointe métallique (en argent) d'une surface d'Ag(111). À chaque étape de cette approche nous avons mesuré la conductance $G$ de la jonction STM (fig. 4a), ainsi que le spectre de la lumière émise (fig. 4b). Le point de contact entre pointe et surface apparait comme une inflexion dans la courbe en (a) et révèle une conductance d'un quantum de conductance $\left(G_{0}=2 e^{2} / h\right)$. À ce point, un seul atome d'argent fait la connexion entre pointe et surface.

Intéressons-nous maintenant à la variation de l'intensité lumineuse lors de l'approche de la pointe (fig. 4c). Pour les faibles conductances, l'intensité de la lumière augmente linéairement avec G. Cet effet est en accord avec le modèle décrit plus haut, et montre que l'émission d'un photon est liée au passage d'un électron dans la jonction. Pour $G \geq 0,1 G_{0}$, un écart à la dépendance linéaire est visible, qui se poursuit par une réduction de l'intensité lumineuse à proximité du contact. Notons que pour ces valeurs de conductance, nous observons donc une diminution de l'émission alors même que le courant tunnel augmente ! ’̀ $G \approx G_{0}$, point de contact, un minimum d'émission apparait.

Ces observations ne s'expliquent pas dans le cadre du modèle de la règle d'or de Fermi. Pour aller au-delà de ce modèle, il est nécessaire de prendre en compte un aspect du courant traversant la jonction que nous avons jusque-là négligé : le bruit de grenaille. Celui-ci est lié à la nature corpusculaire des électrons. Le temps séparant le passage de deux charges au travers de la jonction fluctue, alors même que le courant moyen est constant. Ces fluctuations intrinsèques de courant sont assimilables à un bruit, appelé bruit de grenaille. Nous avons déterminé l'évolution de ce bruit (ligne rouge, fig. $4 d$ ) pour une jonction $\mathrm{Ag} / \mathrm{Ag}$. 
On remarque une très forte similitude entre le rendement quantique d'émission (le rapport entre le nombre de photons et le nombre d'électrons) et la courbe caractérisant l'évolution du bruit de grenaille (ligne rouge, fig. 4d).

On peut formuler l'hypothèse que le bruit de grenaille se comporte ici comme un dipôle oscillant générant un champ électromagnétique. Lorsque ce bruit diminue, l'intensité lumineuse en fait autant. À ce jour, il n'y a pas de description formelle du processus dans les termes que nous venons de présenter. On voit notamment que le terme source de l'expression caractérisant l'émission de photons (expression (1), p. 6) doit être modifié afin de prendre en compte la réduction du bruit de grenaille aux fortes conductances.

Cette expérience constitue également une nouvelle méthode permettant de sonder le bruit de grenaille. Elle devrait permettre de tester la validité des prédictions sur l'évolution de ce bruit à des fréquences (optiques, $\sim 10^{15} \mathrm{~Hz}$ ) jamais atteintes auparavant. Enfin, à l'instar des autres méthodes permettant de sonder le bruit de grenaille d'un conducteur quantique, l'intensité des photons émis apporte des informations sur le nombre et le caractère ouvert ou fermé des canaux de conductance de la jonction.

\section{Conclusions et perspectives}

Les principaux éléments permettant de comprendre l'émission de photons par effet tunnel inélastique sous pointe STM ont été mis en évidence. En accord avec la règle d'or de Fermi, la transition inélastique dans la barrière tunnel est influencée par la densité locale d'états électroniques et par des modes de plasmons localisés sous la pointe STM ou propagatifs sur la surface. Enfin, les fluctuations du courant tunnel jouent un rôle important dans le mécanisme d'excitation des plasmons.

Si de nombreux aspects fondamentaux relatifs à cette émission restent à élucider, la possibilité d'exciter des plasmons de surface avec la pointe d'un STM offre des perspectives uniques. La plupart des études de plasmonique sont actuellement réalisées par excitation photonique avec une résolution spatiale limitée par la diffraction. Avec le STM, on a une méthode d'excitation électrique de basse énergie et de résolution atomique. Suivant le type de modes de plasmons excités, différentes études peuvent être initiées. Avec les modes de plasmons localisés sous la pointe STM, il devient possible d'exciter électroniquement un nano-objet individuel placé dans la nanocavité plasmonique constituée par la jonction tunnel. On peut ainsi activer la luminescence d'un émetteur quantique individuel. Avec les modes de plasmons propagatifs, on peut expérimenter le transfert d'énergie et/ou d'information le long de nanostructures plasmoniques. Dans ce dernier cas, la pointe du STM est utilisée pour exciter un plasmon propagatif à une extrémité de la nanostructure, et la propagation du plasmon vers l'autre extrémité permet d'exciter électroniquement à distance un nano-objet individuel couplé à la nanostructure plasmonique.

Ainsi, l'excitation électrique locale de plasmons avec le STM permet d'initier un nouveau domaine de la nanoélectronique et de la nanophotonique, en réalisant la transformation de signaux électroniques en signaux optiques à l'échelle atomique.

\section{En savoir plus}

- G. Schull, M. Becker et R. Berndt, Phys. Rev. Lett. $101(2008) 136801$.

- L. Douillard et F. Charra, J. Phys. D: Appl. Phys. 44 (2011) 464002.

- T. Wang, E. Boer-Duchemin, Y. Zhang, G. Comtet et G. Dujardin, Nanotechnology 22 (2011) 175201.

- N.L. Schneider, G. Schull et R. Berndt, Phys. Rev. Lett. 105 (2010) 026601 